\title{
A Model of Adaptive Accountability for Innovations in an
}

\author{
Academic Institution \\ Abu-Hussain Jamal ${ }^{1}$, Oleg Tilchin ${ }^{1}$ \\ ${ }^{1}$ Computer Science Department, Al-Qasemi Academic College of Education, Baqa El-Gharbieh, Israel \\ Correspondence: Oleg Tilchin, Computer Science Department, Al-Qasemi Academic College of Education, Baqa \\ El-Gharbieh, Israel.
}

Received: February 25, 2019

Accepted: March 4, 2019 Online Published: March 26, 2019

doi:10.11114/bms.v5i1.4174

URL: https://doi.org/10.11114/bms.v5i1.4174

\begin{abstract}
An academic institution has to encourage an innovation activity in order to meet the requirements of a modern, dynamic, and competitive environment. The innovation activity of the institution is engendered by instructors' innovative intentions to create and assess the innovation proposals. However, there are challenges that prevents realization of the innovative intentions. The challenges are caused by lack a model that would shape process of revealing the effective proposals and building accountability for their development. The ASVA model including components "Accountability", "Submission", "Valuation", and "Awarding" is suggested. The model provides: creation and assessment of the innovation proposals by the instructors, determination of the instructors' accountability measures for development of the innovation proposals, adaptation of the instructors' accountability measures to the values of the innovation proposals, revelation of the key instructors who are creators the most valuable innovation proposals for attaining the current aim of the institution, formation of flexible collaboration groups by taking into consideration of willingness and ability of the proposal's valuators to share accountability for the innovation proposal development with the proposal creator, and adjustment of the size of award received by the instructors to the values of the innovation proposals.
\end{abstract}

Keywords: adaptive accountability, innovation

\section{Introduction}

An academic institution should choice the innovation-based activity to remain viable in a changed and competitive environment (Tierney \& Lanford, 2016, Kaplan, 2017). The innovation activity guided by leaders and managers comprises the following basic stages: creation the innovation proposals for improvement of the institution performance and development of the innovations on the basis of these proposals (Poole et al., 2000; Jones, 2012).

However, there are challenges which obstruct the innovation activity. The challenges are: the institution culture doesn't promote innovations; the institution leadership and management can't reveal the main directions of the innovation activity are supported by available knowledge and skills of instructors; the adjustment to the changed objectives of the institution is missing; wide involvement of the instructors in the innovation activity isn't provided; the instructors' innovation activity is not stimulated and motivated; there isn't productive collaboration of the instructors through all levels of the institution structure; the driving power of accountability of the instructors for changes isn't used.

A favorable environment in the institution should be formed for solving these challenges and promoting the innovation activity of the instructors (Lawson \& Samson, 2001; Horibe, 2009). The basic elements of the environment are motivation of the instructors, assessment of their innovation activity, the instructors' collaboration, and accountability of the instructors for the innovation activity.

The motivation engenders the instructors' desire to participate in innovation activity and can be realized by the appropriate reward mechanisms (Nacinovic et al., 2009; Glasberg \& Ouerghemi, 2011). The assessment of the instructors' innovation activity has to be the effective instrument providing a choice of the qualitative innovation proposals. The instructors' collaboration groups are formed for development of the innovations. Collaboration should be adapted to peculiarity of the innovations and unlimited by the institution structure.

The accountability provides the instructors of significance of the institution' goal (Evans, 2008). The instructors' accountabilities should be based on their desires and abilities to develop the innovations for attainment of the 
institutional effectiveness (Ewell, 2011). A flexible mechanism of determining accountability according to changing the directions of the innovation activity is needed. Furthermore, taking and sharing accountability of the instructors for development of the innovations should be encouraged.

However, the elements forming the favorable environment in the institution and the suitable order of their use for promoting the innovation activity of the instructors are not developed. Therefore, the goal of this paper is to introduce a systematic model providing a framework for elaboration, integration and ordered use of the favorable environment elements for promotion of the innovation activity in the academic institution.

The model provides: shaping the process of motivated creation and peer assessment of the innovation proposals; determination of the instructors' accountability measures for development of the innovations; forming the instructors' collaboration groups, composition of which aligned with the specificity of the innovation proposals and the measures of the instructors' shared-accountability for their development ; unfolding leading innovation directions and the key instructors of the innovation activity.

\section{Related Research}

The research contributed to promotion of the instructors' innovation activity in the academic institution are examined. Jakovljevic (2018) created the ORED model (observation, revelation, exploration, design) for empowerment and development of innovations in an academic institution. The model takes into account of the innovators' individual abilities, their innovation development cycles, and the individual and institutional dynamics in the innovative process. Findlow (2008) explored contradiction between bureaucratic models of higher education accountability and academic innovation activity. The author presented a consideration about how to promote the innovations and provide accountability for their development with such contradiction. The consideration is based on the innovators' experiences in the certain institution.

Ayers (2005) claimed the organizational environment should be formed allowing to realize desire and reediness of the employees to take accountability for their work. Martins E. \& Martins N. (2002) determined the elements of the organizational environment promoting the innovation development. The elements are the innovative strategy, a dynamic group-based organizational structure allowing freedom of the employees' interactions, recognition and reward inducing the innovation activity, and the communication capabilities.

Brands \& Kleinman (2010) developed the procedures promoting improvement of organizational performance through innovation. The procedures are creating the suitable values of the organizational culture, building accountability, idea management, watching, measuring, and rewarding. Evans (2008) declared improvement of organizational performance is resulted by its strategy taking into consideration of accountability and building relationships among group members on the basis of accountability.

Kennedy \& Schleifer (2006) affirmed the organization's hierarchical structure should be replaced by the team-based structure in order to empower collaborative and accountable development of innovations. Ettlie (2011) elaborated the innovation process and emphasized the constructive role of collaboration in this process.

Glasberg \& Ouerghemi (2011) considered the motivation of employees as one of the basic elements promoting development of innovation in an organization. Nacinovic et al. (2009) believed promotion of the innovation activity in an organization can be provided by applying of the suitable reward system.

Chang et al. (2017) explored influence of accountability for process, result, and hybrid (process and result) on adaptive performance supporting change of organizational strategies for attaining the better results. The authors demonstrated accountability for process strengthened adaptive performance in the short-term but inhibited adaptive performance in the long-term. Accountability for result forced adaptive performance in the long-term. Hybrid accountability caused flexibility in transition from existing strategy to new one. Green et al. (2000) discovered hybrid accountability and reward systems caused comprehensive and flexible thinking.

The analysis of the above publications allows to conclude the challenges caused by lack of a model promoting the innovation activity in the academic institution remain. The favorable environment for innovations combining such elements as motivation, evaluation, accountability, and collaboration hasn't be described. The values of the innovation proposals through peer assessment hasn't be determined. Building accountability of instructors for development of the innovation proposals hasn't be realized. Adaptation of the measures of accountability for development of the innovation proposals to their values hasn't be provided. Forming dynamic collaboration groups caused by aspiration of the proposals' valuators to share accountability for development of the proposals with their creators hasn't be considered. The motivation mechanism ensuring a reward for all participants of creation and assessment of the innovation proposals hasn't be presented. Aligning the size of the rewards with the innovation proposals' values hasn't be performed. 


\section{The Model of Adaptive Accountability for Innovations in the Academic Institution}

The purpose of the model is promotion of the innovation activity in the academic institution. It is attained by creation and assessment of the innovation proposals by the instructors, determination and realization of the instructors' intentions to take or share accountabilities for development of the innovations on the basis of the proposals, adaptation of taking and sharing accountability of the instructors for developing the innovations to the proposals' values, use of a flexible encouragement mechanism for inducing the instructors to the innovation activity, and aligning compensations received by the instructors with the proposals' values.

Creation and peer evaluation of innovative proposals is guided by the institution goal, a submission frame, and the encouragement mechanism. Manifestation of the innovation proposals motivates the instructors to create their own proposals or to participate in development of the presented proposals. It leads to wide involvement of the instructors in the innovation activity. Alignment of the instructors' compensations with the proposals' values enables reasonable reward.

The intention of the proposal's creator to take accountability for its development is evaluated by peers. The instructor's intention to share accountability for performance of the innovation is resulted by self-examination of his/her capability to support the proposal development and evaluation of the proposal creator. It leads to creation of the adaptive collaborative groups unlimited by the institution structure. Thereby, productive collaboration is organized.

Adaptation of taking accountability of the instructors for developing the innovation proposals to their values is provided by aligning the accountability measures with the proposal values.

The ASVA model includes the following interconnected model components: Accountability, Submission, Valuation, and Awarding (Fig.1). The order of interaction of the model components is showed by arrows. The "Accountability" is the central component of the ASVA model. It creates adaptive accountability of the instructors for development of the innovation proposals.

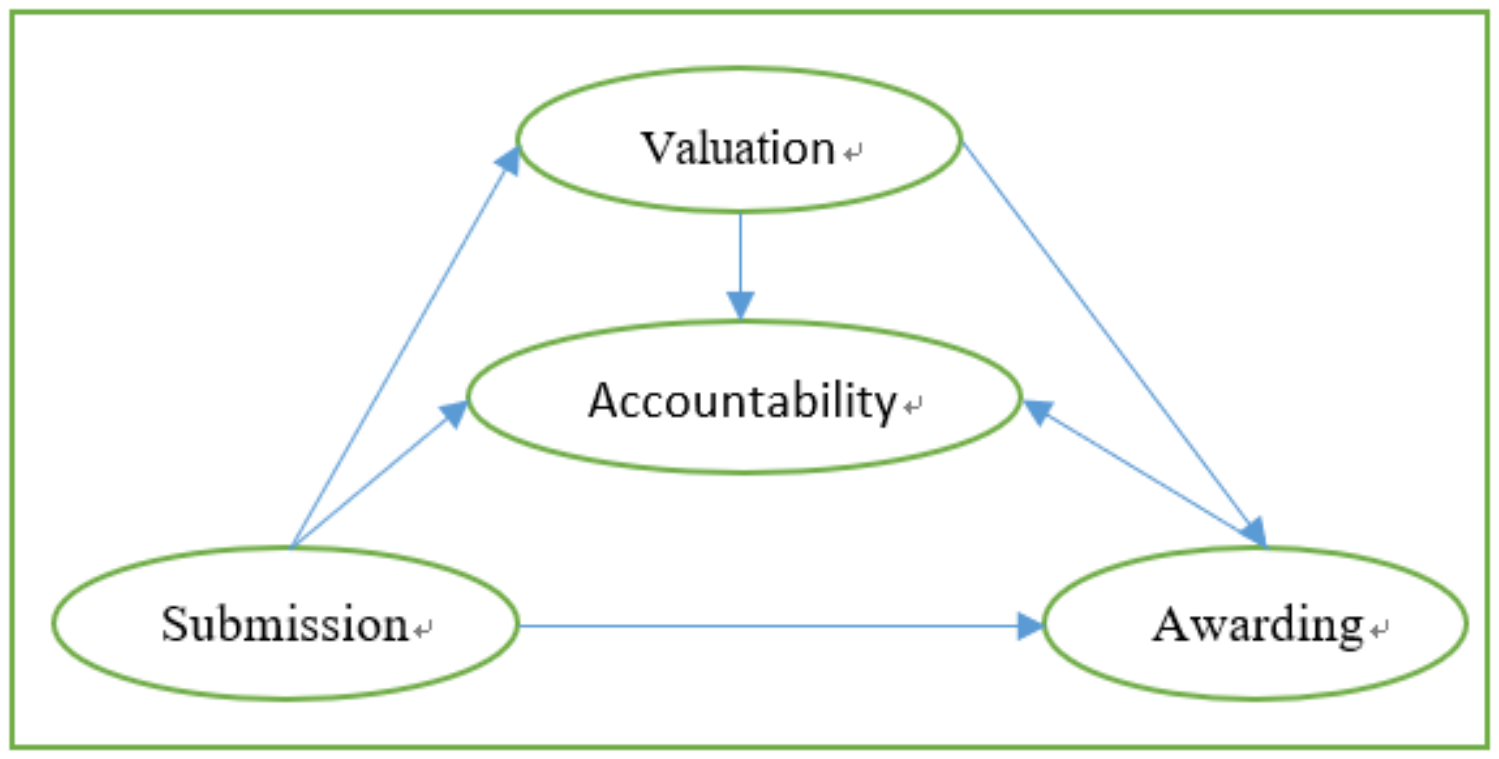

Figure 1 . The adaptive accountability model

The "Submission" component.

The objective of the "Submission" component is to promote submission and evaluation of the innovation proposals by the instructors regarding the innovative purpose of the institution.

The procedures of the "Submission" component are: the innovative purpose setting, determining the possible roles of the instructors in creation and assessment of the innovation proposals, forming requirements for submission of the innovation proposals, and defining the reward conditions.

The innovative purpose setting consists in determining the purpose that should be attained as a result of the innovation activity, creating a list of the innovative directions (the directions may not be specified if the managers want to reveal the new directions are cultivated by instructors), and informing the instructors about the purpose.

The possible roles of an instructor in creation and assessment of the innovation proposals are a proposal-creator, a proposal-valuator, and a potential collaborator. The proposal-creator creates the proposal; the proposal-valuator 
assesses the proposal, the potential collaborator is the valuator who intends to collaborate while development of the innovation based on the proposal.

The submission the innovation proposals by the instructors is adjusted by requirements. The requirements need is caused the aspiration to provide the qualitative proposals. The requirements are: the size of a proposal is limited; the proposal must be structured regarding the following elements: objectives, originality, essence, and expected results; the proposal can be concerned with only one innovative direction (if the directions are specified); the instructor may submit only one proposal for the specified direction; the number of proposals that may be submitted by an instructor for a few directions and the total number of proposals that may be submitted by an instructor are limited.

The reward conditions are aimed at inducing the instructors to create and assess the innovation proposals, and to intensify the collaborative innovative activity. The reward conditions are: all instructors participating in submitting and assessing the innovation proposals receive the reward; the reward for each proposal is divided between the creator and the valuators of the proposal according to the reward percentage depending on the current situation in the institution; the most reward corresponds with the proposal having the most peer or management assessment; size of the personal reward depends on the determined values of his (her) proposals and the values of peer proposals which he (she) assesses.

The "Valuation" component.

The objectives of the "Valuation" component are determining the values of the innovation proposals, finding the leading direction of innovation activity, and revealing the key instructors to achievement of the innovative purpose.

The value of each proposal is determined as a result of its assessment by peers or managers (if the proposal isn't evaluated by instructors), examination of the valuators by the creator and self-evaluation of possibility of the valuators to collaborate while development of the proposal.

The procedures of the "Valuation" component are introducing the assessment restrictions, realizing the instructor's assessment of the innovation proposal, calculating the total assessment of the proposal, assessing potential collaboration of the instructors, determining the value of the proposal, finding the leading direction of the innovation activity, and revealing the basic and key instructors.

Qualitative assessment of the proposals and effective collaboration while their performance can be provided due to the following restrictions: the proposal-creator may not be the valuator of peer proposals concerning the innovative directions to which his (her) proposals belong; the restricted number of proposals may be assessed by every instructor, and the number of proposals for which the instructor may be the potential collaborator is limited.

The instructor's assessment of the innovation proposal is determined by formula:

$$
\mathrm{a}(\mathrm{p})=\sum_{\mathrm{i}=1}^{\mathrm{n}_{\mathrm{i}}} \mathrm{w}_{\mathrm{i}} * \mathrm{r}_{\mathrm{i}} / 100
$$

where

$a(p)$ is the proposal assessment,

$\mathrm{w}_{\mathrm{i}}$ is the weight of $\mathrm{i}$ element of the innovation proposal structure, $\mathrm{n}$ is the number of the structure elements,

$r_{i}$ is the assessment rating for $i$ element of the proposal structure, $i=1, \ldots, n$, the ratings are set by the instructor.

The weights of the proposal structure elements are set by managers depending on specificity of the innovative purpose. The established range for assessment is set from 0 to 5 . The total assessment of the proposal is calculated as sum of the assessments performed by the instructors.

Example1:

The instructor's assessment of the innovation proposal calculated by formula (1) is represented by Table1. This assessment is 3.3 .

Table 1. The instructor's assessment of the innovation proposal

\begin{tabular}{|c|c|c|c|}
\hline $\begin{array}{l}\text { The elements of the innovation } \\
\text { proposal structure }\end{array}$ & $\begin{array}{l}\text { The weights of the proposal } \\
\text { structure elements (\%) }\end{array}$ & $\begin{array}{l}\text { The assessment } \\
\text { range }(0-5)\end{array}$ & The assessment value \\
\hline Objectives & 20 & 1 & 0.2 \\
\hline Originality & 25 & 5 & 1.25 \\
\hline Essence & 35 & 3 & 1.05 \\
\hline Results & 20 & 4 & 0.80 \\
\hline The assessment of the proposal & & & 3.30 \\
\hline
\end{tabular}


Assessments of the proposal by four instructors are 2.7, 3.3, 3.7, and 4.3, accordingly. Then, the total assessment of the proposal is equal to 14 .

The assessment of potential collaboration of the instructors while development of the innovation proposal can be determined. The assessment of collaboration for proposal having the maximal number of potential collaborators is set as a fixed value. The assessment of collaboration for each from others proposals is determined relative to the fixed value pro rata the number of the collaborators.

Example2:

There are four proposals. The number of the potential collaborators for their development equals 5, 4, 3, and 2, accordingly. The maximal number of collaborators equals 5. This value corresponds to the first proposal. Hence, the assessment of potential collaboration while developing this proposal is set equal to 5 . Then, the assessments of potential collaboration for others proposals are equal to 4,3 , and 2 , accordingly.

The value of the innovation proposal can be determined as the sum the total assessment of the proposal and assessment of potential collaboration while its development.

Example3:

The total assessment of the proposal equals 14 (Example1) and the assessment of potential collaboration while developing this proposal equals 4 (Example 2). Then, the proposal value equals 18.

The basic creators of the innovation proposals regarding the innovative directions, the leading direction, and the key creator can be revealed. At first, the basic creators regarding the innovative directions are revealed. They are the creators of the proposals having the maximal values in each from the direction. Then, the value of each direction is calculated. It is sum of values of the proposals presented for the direction. Next, the leading direction (directions) can be determined. It is direction having the maximal value. At last, the key creator is determined. He or she is the basic creator for the leading direction.

Example4:

There are two innovative directions. There are four proposals for each direction. The values of the proposals presented relative to the first direction are equal to $9,10,11$, and 20, accordingly. The values of the proposals presented regarding the second direction are equal to $7,8,9$, and 10 , accordingly. Then, the basic creator for the first direction is the creator of the proposal with the value equal to 20 , and the basic creator for the second direction is the creator of the proposal having the value equal to 10 .

Next, the values of these directions are calculated by summation of the corresponding proposals values. Hence, the direction values are equal to 50 and 34, accordingly. Comparison of these values allows for the conclusion that the first direction must be chosen as the leading direction. At last, the key creator is revealed. He or she is the basic proposal-creator for the leading first direction.

The "Awarding" component.

The objective of the "Awarding" component is determining rewards received by the instructors for creation and assessment of the proposals. The rewards is aligned with the proposal values.

The procedures of the "Awarding" component are calculation of the award for all instructors regarding each from the innovation directions; determination of the award for instructors related to each innovation proposal; defining divided award for creation and assessment of the proposals according to the adjusted reward percentage; calculation of the personal award for every instructor participating in creation and assessment of the innovation proposals.

The award regarding each innovation direction is determined by dividing the award fund on the parts pro rata of the direction value. The award for each innovation proposal is calculated by dividing the reward concerning to the innovative direction pro rata the proposal value.

The reward percentages for divided award of the instructors for creation and assessment of the innovation proposals serve as a means for inducing creation of the proposal, improving quality of the proposal assessment, and supporting collaboration while development of the proposals. The award for each proposal is divided on the awards related to creation and assessment. The awards are calculated on the basis of the reward percentages.

The equal award is set for the proposal-valuators. Consequently, the award for every proposal-valuator is calculated by dividing the award for assessment of the proposal on the number of the proposal-valuators.

The personal award for every instructor is determined by taking into account his or her activity concerning creation and assessment of the innovation proposals. 
Example 5:

The award fund is equal to $\$ 1680$. There are two innovation directions. The values of the directions are equal to 50 and 34, accordingly (Example 4). Then, the awards for the instructors regarding the first and the second directions are equal to $\$ 1000$, and $\$ 680$, accordingly.

There are four proposals in each from the directions. The values of the proposals belonging to the first direction are equal to $9,10,11$, and 20, accordingly. The values of the proposals belonging to the second direction are equal to $7,8,9$, and 10, accordingly. Then, the awards for creation and assessment of the proposals from the first direction are equal to $\$ 180, \$ 200, \$ 220$, and $\$ 400$, accordingly. Similarly, the awards for creation and assessment of the proposals from the second direction are equal to $\$ 140, \$ 160, \$ 180$, and $\$ 200$, accordingly.

The reward percentages for creation and assessment of the proposals are set equal to $60 \%$ and $40 \%$, accordingly. Then, the rewards for the creators of the proposals belonging to the first direction are equal to $\$ 108, \$ 120, \$ 132$, and $\$ 240$, accordingly. Hence, the rewards for valuators of the proposals belonging to the first direction are $\$ 72, \$ 80, \$ 88$, and $\$ 160$, accordingly.

Similarly, the rewards for the creation and assessment of the proposals belonging to the second direction can be calculated. So, the reward for the creator of the fourth proposal is equal to $\$ 120$. Then, the reward for the valuators of this proposal is equal to $\$ 80$ since the award for creation and assessment of this proposal is equal to $\$ 200$. There are two valuators of this proposal. Hence, the award for every valuator of the proposal is equal to $\$ 40$.

The instructor is creator of the second proposal belonging to the first direction. This instructor is also the valuator of the fourth proposal belonging to the second direction. Then, the award the instructor as the creator is equal to $\$ 120$ and him or her award as the valuator is equal to $\$ 40$. Because of that, the personal award of the instructor for creation and assessment of the proposals is equal to $\$ 160$.

The "Accountability" component.

The objective of the "Accountability" component is to build adaptive accountability of the instructors for development of the innovation proposals.

The "Accountability" component provides adaptation taking and sharing accountability of the instructors for development of the innovation proposals to their values. It is realized by adjustment of the measures of accountability for development of the proposals to the proposal values.

The procedures of the "Accountability" component are setting the measure of joint accountability for the proposal-creator and the potential collaborators for development of the proposal, setting the accountability measures for the proposal-creators, determining the accountability measures of the potential collaborators, determining the accountability measure of the potential collaborator.

The measure of joint accountability of the proposal-creator and the potential collaborators for developing the proposal is set equal to the proposal value. The more accountability measure of the instructors corresponds with the more value of the proposal. Thereby, adaptation of taking accountability of the instructors for performing the innovation proposals to the values of the innovative proposals is provided.

The joint accountability measure is shared between creator and the potential collaborators. The accountability measure of the proposal-creator is set equal to the total assessment of the proposal. The measure of accountability of the basic creator for the innovation direction is more than the measure of accountability each from creators others proposals regarding this direction. The accountability measure of key creator is the most.

The accountability measure of the potential collaborators is set equal to the assessment of potential collaboration while development of the proposal. According to aforementioned, the assessment of potential collaboration depends on the number of the potential collaborators. Consequently, the accountability measure for development of the proposal having the more number of the potential collaborators is more than the accountability measure for development of the proposal with the less number of the potential collaborators.

The accountability measure of the potential collaborators while development of the innovation proposal is shared among them. Consequently, the accountability measure of the potential collaborator while development of the proposal is determined by formula:

where

$$
\mu_{\mathrm{col}}=\mathrm{a}\left(\mathrm{p}_{\mathrm{col}}\right) / \mathrm{m}
$$

$\mu_{\mathrm{col}}$ is the measure of accountability of the potential collaborator,

$\mathrm{a}\left(\mathrm{p}_{\mathrm{col}}\right)$ is the assessment of potential collaboration while developing the proposal, 
$\mathrm{m}$ is the number of the potential collaborators.

Example 6:

The measure of joint accountability of the creator and the potential collaborators while developing the innovation proposal is set equal to18 since the proposal value equals 18 (Example3).

The total assessment of the proposal equals 14 (Example1). Hence, the accountability measure of the proposal-creator equals 14.

The assessment of potential collaboration while development of the proposal equals 4 (Example 2). Hence, the accountability measure of the potential collaborators is set equal to 4 . The measure of accountability of the potential collaborator is equal to 1 , because of both assessment of potential collaboration is equal to 4 and the number of the collaborators equals 4 (formula2).

\section{Conclusion}

The suggested ASVA model shapes process submission of innovation proposal, their evaluation, awarding the instructors, and building the instructors' accountability for development innovations on the basis of proposals in an academic institution.

The accountability measures are aligned with the innovation proposal values that are determined as a result of submission of the proposals and their peer assessment. Due to that, adaptation of accountability to the proposal values is attained.

Building accountability engenders dynamic collaborative groups. The members of each group have intention to share accountability for development of the innovation proposal with the proposal-creator.

Aspiration of the instructors to create the qualitative proposals and take or share accountability for their development is encouraged by reward system. It ensures adaptation of the instructors' rewards to the innovation proposal values.

The ASVA model allows: creating the dynamic and innovative environment in the institution; forming an institution culture including an accountability culture, a culture of learning, and a culture of collaboration contributing to the balance between competition and collaboration; stimulating and facilitating knowledge creation and sharing; providing exchange of information among instructors regarding their interests and preferences; reasonable taking accountability by instructors for developing innovations; and revealing well-founded innovative directions and the key instructors.

The ASVA model can serve as the constructive tool providing significant improvement in the institution performance due to promoting the intensive and fruitful innovation activity of the instructors.

\section{References}

Ayers, K. (2005). Creating a responsible workplace. HR Magazine, 50(2), 1-3. http://www.engagementisnotenough.com/pdfs/HR_Magazine_RBW.pdf

Brands, R. F., \& Kleinman, M. J. (2010). Robert's Rules of Innovation: A 10-Step Program for Corporate Survival, (1st ed.). New Jersey, NJ: Wiley.

Chang, W., Atanasov, P., Patil, S., Mellers, B. A., \& Tetlock, P. E. (2017). Accountability and adaptive performance under uncertainty: A long-term view. Judgment and Decision Making, 12(6), 610-626.

Evans, H. J. (2008). Winning with Accountability: The Secret Language of High-Performing Organizations, (6 ed.). Dallas, TX: Corner Stone Leadership Institute.

Ewell, P. T. (2011). Accountability and institutional effectiveness in the community college, In: New directions for community colleges, N153, New Jersey, NJ: Willey Periodicals Inc. https://onlinelibrary.wiley.com/doi/pdf/10.1002/cc.434

Findlow, S. (2008). Accountability and innovation in higher education: A disabling tension? Studies in Higher Education, 33(3):313-329. https://doi.org/10.1080/03075070802049285

Glasberg, R., \& Ouerghemi, K. (2011). Innovation in Human Resources. A Theoretical Advancement on Employee Motivation and Organizational Innovation, 2nd International Conference on Economics. Business and Management, IPEDR, 22, IACSIT Press, Singapore.

Green, M. C., Visser, P. S., \& Tetlock, P. E. (2000). Coping with accountability cross-pressures: Low-effort evasive tactics and high-effort quests for complex compromises. Personality and Social Psychology Bulletin, 26(11), 1380-1391. https://doi.org/10.1177/0146167200263006

Horibe, F. (2009). Creating the Innovation Culture: Leveraging Visionaries, Dissenters and Other Useful Troublemakers, (1st ed.). New Jersey, NJ: Wiley 
Jakovljevic, M. (2018). A model for innovation in higher education. South African Journal of Higher Education, 32(4),1-23. https://doi.org/10.20853/32-4-2432

Jones, T. (2012). On Innovation, New York, NY: Essential Ideas, Inc.

Kaplan, S. (2017). The Invisible Advantage: How to Create a Culture of Innovation, Austin, TX: Greenleaf Book Group Press.

Kennedy, F. A., \& Schleifer, L. (2006). Innovation: Achieving Balance among Empowerment, Accountability and Control, In Michael M. Beyerlein, Susan T. Beyerlein, Frances A. Kennedy (ed.) Innovation through Collaboration (Advances in Interdisciplinary Studies of Work Teams, 12) 113 - 135, Bingley, West Yorkshire, England: Emerald Group Publishing Limited.

Lawson, B., \& Samson, D. (2001). Developing Innovation Capability in Organizations: A Dynamic Capabilities Approach. International Journal of Innovation Management, 5(3), 377-400. https://doi.org/10.1142/S1363919601000427

Martins, E., \& Martins, N. (2002). An Organizational Culture Model to Promote Creativity and Innovation. Journal of Industrial Psychology, 28(4), 58-65. https://doi.org/10.4102/sajip.v28i4.71

Nacinovic, N., Galetic, L., \& Cavlek, N. (2009). Corporate culture and innovation: implications for reward systems. World Academy of Science, Engineering and Technology, 397-402. http://www.waset.org/publications/8755

Poole, M. S., Van de Ven, A. H., Dooley, K., \& Holmes, M. E. (2000). Organizational Change and Innovation Processes: Theory and Methods for Research, Oxford, England, UK: Oxford University Press.

Tierney, W. G., \& Lanford, M. (2016). Conceptualizing Innovation in Higher Education, In M.B. Paulsen (ed.), Higher Education: Handbook of Theory and Research, New York, NY: Springer International Publishing.

\section{Copyrights}

Copyright for this article is retained by the author(s), with first publication rights granted to the journal.

This is an open-access article distributed under the terms and conditions of the Creative Commons Attribution license which permits unrestricted use, distribution, and reproduction in any medium, provided the original work is properly cited. 